\title{
Genomic Sequence Resource of Alternaria alternata Strain B3 Causing Leaf Blight on Ophiopogon japonicus
}

\author{
Mingshuang Wang, ${ }^{1,2}$ Xin Hou, ${ }^{3}$ and Huizhong Wang ${ }^{1,2, \dagger}$ \\ ${ }^{1}$ College of Life and Environmental Sciences, Hangzhou Normal University, Hangzhou 310036, China \\ ${ }^{2}$ Zhejiang Provincial Key Laboratory for Genetic Improvement and Quality Control of Medicinal Plants, \\ Hangzhou Normal University, Hangzhou 310036, China \\ ${ }^{3}$ College of Plant Protection, Shandong Agricultural University, Shandong Key Laboratory of Agricultural \\ Microbiology, Tai'an 271018, China
}

\begin{abstract}
Black spot of Ophiopogon japonicus is an economically important disease, which can result in significant losses of both yield and quality of this traditional Chinese medicinal plant. The disease is caused by the small-spored fungal pathogen Alternaria alternata, a necrotrophic fungus that is ubiquitously distributed in the environment. Here, we present the draft wholegenome sequence of $A$. alternata strain B3 that caused black spot on $O$. japonicus. The assembly consists of 76 contigs with an estimated genome size of $33.8 \mathrm{Mb}$. Furthermore, we identified genes that may be associated with the pathogenicity, such as carbohydrateactive enzymes, secreted proteins, and secondary metabolite gene clusters. This genome resource will provide a useful source for future research on the evolution of pathogenicity of A. alternata and phylogenomic analysis in delineating phylogenetic lineages within the genus Alternaria.
\end{abstract}

Alternaria alternata, one of the small-spored Alternaria spp., can be ubiquitously found in soil, various plants, and decaying plant debris. Some strains in this species can cause diseases on more than 400 host plants and result in severe crop losses worldwide (Thomma 2003). However, the systematics of delimitation of $A$. alternata has been ambiguous. Some Alternaria species can be morphologically very similar but exhibit substantial pathological differences, and researchers failed to delineate significant variation among those species based on DNA sequences of popular marker genes (Woudenberg et al. 2013, 2015). Therefore, the concept of formae speciales and pathotypes were introduced into the systematics of $A$. alternata (Tsuge et al. 2013; Woudenberg et al. 2015). For example, the pathogen that causes citrus brown spots on tangerines and tangerine hybrids was defined as the tangerine pathotype of $A$. alternata (Wang et al. 2019).

Ophiopogon japonicus (Thunb.) Ker-Gawl (Liliaceae) is a traditional Chinese medicinal plant widely cultured in China, and its root, which was admitted as one of the functional food ingredients by the Ministry of Health of the People's Republic of China, is used as the main ingredient in many prescriptions. Over thousands of years, $O$. japonicus has shown curative effects on symptoms such as cough and phlegm (Chen et al. 2016). However, black spot caused by $A$. alternata is an important leaf disease during $O$. japonicus cultivation. At the early stage of infection, yellow or brown patches form at the apex of leaves, which then proceed toward the leaf base; at later stages, the spots turn black and putrescent. The disease rate can reach up to $90 \%$ in years with high temperatures and heavy rainfall, which seriously affects the yield and quality of $O$. japonicus.

${ }^{\dagger}$ Corresponding author: H. Z. Wang; whz62@163.com

The author(s) declare no conflict of interest.

Accepted for publication 27 August 2020
Funding

This work was supported by the Major Increase or Decrease Program in the Central Finance Level (2060302),

Zhejiang Provincial Key Research \&

Development Project Grants

(2018C02030), and Hangzhou

Scientific and Technological Program (20191203B06).

\section{Keywords}

black spot, Ophiopogon japonicus, Alternaria alternata, genomics, pathogenicity 
Table 1. Genome statistics of Alternaria alternata strain B3.

\begin{tabular}{lc} 
Features & Alternaria alternata B3 \\
Genome size (Mb) & 33.8 \\
BUSCOs (\%) & 99.7 \\
Number of contigs & 76 \\
N50 (Mb) & 1.48 \\
N90 (Mb) & 0.48 \\
GC content (\%) & 51.0 \\
Protein-coding genes & 12,680 \\
Gene density (number of genes per Mb) & 374 \\
Average intron number & 2 \\
Average intron length (bp) & 76 \\
Average exon number & 3 \\
Average exon length (bp) & 500 \\
Mean gene length (bp) & 1,663 \\
Number of genes with intron & 10,121 \\
tRNA genes & 141 \\
Repeat content (\%) & 1.10 \\
Carbohydrate-active enzymes & 538 \\
Secreted proteins & 1,049 \\
Mall secreted cysteine-rich proteins & 186 \\
Secondary metabolites gene clusters & 26 \\
\hline
\end{tabular}

The mechanisms underlying the ability of $A$. alternata to effectively infect $O$. japonicus leaves and its interaction with host cells remain completely unknown. The genes involved in sporulation, stress response, nutrient absorption, and toxin biosynthesis of this pathogen are yet to be identified. For these reasons, we have fully sequenced the genome of the B3 strain of $A$. alternata that caused black spots on $O$. japonicus.

Genome sequencing and assembly. A total of eight $A$. alternata strains were isolated from blight infected leaves from Zhejiang Province, China. These isolates showed identical morphological characteristics and we randomly chose strain B3 as a representative strain. This strain was ascertained as the causal agent of leaf blight of Ophiopogon japonicus using Koch's postulates by inoculating it on healthy $O$. japonicus leaves and reisolating it from the inoculated leaves. Cultures of this fungal strain were maintained in liquid potato dextrose broth (PDB, extract of $200 \mathrm{~g}$ potato-boiled water and $20 \mathrm{~g}$ dextrose per liter) on a rotary shaker at $160 \mathrm{rpm}$ at $25^{\circ} \mathrm{C}$ for 3 days. The genomic DNA of $A$. alternata strain B3 was extracted from the mycelia with the CTAB method (Möller et al. 1992). Short insert library (350 bp) was constructed and sequenced on an Illumina Hiseq 4000 platform (Nanjing Personalbio Co., Ltd.), generating 150 bp paired-end reads. Raw reads were trimmed of adapter sequences and low-quality reads using Trimmomatic v. 0.38 (Bolger et al. 2014). In total, $3.8 \mathrm{~Gb}$ clean reads were generated in the sequencing process, which corresponds to more than 100 -fold of sequence depth. The genome was de novo assembled into 76 contigs (>1 kb) using SPAdes v 3.14.1 (Bankevich et al. 2012) and resulted in a genome size of $33.8 \mathrm{Mb}$ with an N50 and $\mathrm{N} 90$ of $1.48 \mathrm{Mb}$ and $0.48 \mathrm{Mb}$, respectively (Table 1). The completeness of the genome assembly was estimated by BUSCO v4.0.2 based on lineage-specific profile library dothideomycetes_odb10 (Simão et al. 2015). We found 3,773 out of 3,786 (99.7\%) BUSCO groups in the $A$. alternata strain B3 genome (Table 1), indicating a very high degree of completeness.

The overall $\mathrm{G}+\mathrm{C}$ content of the $A$. alternata strain B3 assembly was $51.0 \%$. The tRNA and its secondary structure were predicted by tRNAscan-SE v 2.0 (Chan and Lowe 2019), revealing 141 tRNA genes (Table 1). Simple repeats, transposable elements, low-complexity sequences, and satellites were detected and annotated using RepeatMasker open 4.1.0 (http://www.repeatmasker.org). Results showed that repetitive sequences represented $1.1 \%$ of the genome (Table 1). The actual repeat content is expected to be larger as small contigs shorter than $1 \mathrm{~kb}$ (we filtered them out) may contain many repetitive sequences.

Genome annotation. To obtain high-quality gene calls, RNA-Seq was conducted with the same sample (mycelia harvested from PDB) and 6.0 Gb Illumina paired-end reads were obtained. RNA-seq data were then aligned to the genome using HISAT v 2.0.9 (Kim et al. 2019). Genome annotation was performed using the BRAKER version 2.0 pipeline (Hoff et al. 2019) based on GeneMark-ES v 4.48 (Borodovsky and Lomsadze 2011) and Augustus v 
2.5.5 (Stanke et al. 2008), combining the RNA-seq-based gene prediction and ab initio gene prediction. A total of 12,680 protein-coding genes were predicted in the $A$. alternata strain B3 genome, with the average gene length $1,663 \mathrm{bp}$ (Table 1). To functionally annotate the 12,680 genes in the $A$. alternata strain B3 genome, we classified their protein products into protein families using the Pfam, v 32.0 databases (El-Gebali et al. 2018). Results showed that 8,903 genes contained Pfam protein domains and they were assigned to 3,916 protein families.

Carbohydrate-active enzymes (CAZymes) play important roles in the breakdown of complex carbohydrates and are responsible for the acquisition of nutrients from plants for plant-associated fungi (Kubicek et al. 2014). The carbohydrate-active enzymes were annotated by the web-based dbCAN2 meta server (supported by $\geq 2$ tools) (Zhang et al. 2018). A total of 538 putative CAZyme genes were identified in $A$. alternata strain B3, which included 270 glycoside hydrolases (GHs), 108 auxiliary activities (AAs), 90 glycosyl transferases (GTs), 39 carbohydrate esterases (CEs), 24 polysaccharide lyases (PLs), and 7 carbohydratebinding modules (CBMs).

Pathogens can secrete a series of proteins that are deployed to the host-pathogen interface during infection, and secretome proteins play an important role in pathogenicity (Lo Presti et al. 2015). Putative extracellular proteins were identified as secreted proteins if they contained a signal peptide, located at the extracellular subcellular, and lacked transmembrane domains and glycosylphosphatidylinositol (GPI)-anchor signals. To identify secreted proteins, we used SingalP 5.0 (Armenteros et al. 2019) and TMHMM v 2.0 (Krogh et al. 2001) to predict the signal peptide and transmembrane domains (PredHel <2) and we excluded nonextracellular and GPI-anchored proteins by using targetP 1.1 (Emanuelsson et al. 2000) and GPI-SOM (Fankhauser and Mäser 2005). Approximately $8 \%$ of the total proteins $(1,049)$ of A. alternata strain B3 were predicted to be secreted. The GO enrichment in molecular functions of the secreted proteins was then produced with the dcGO database (Fang and Gough 2013). "Hydrolase activity" was the most abundant molecular function of the secretome; other GO terms over-represented among the secreted proteins included "exopeptidase activity," "transferase activity, transferring hexosyl groups," "lipase activity," "isomerase activity," "exopeptidase activity," and "UDP-glycosyltransferase activity." All resulting secreted proteins that were shorter than 200 amino acids in length and contained at least four cysteine residues were considered as small secreted cysteine-rich proteins (SSCPs) (Wang et al. 2019). According to this standard, 186 of them were SSCPs and their functions were largely unknown.

Secondary metabolites, especially fungal toxins, are believed to be involved in the pathogenicity of many plant pathogenic fungal species and can be described as potential virulence factors. Fungal secondary metabolite pathways were predicted using the online tool antiSMASH 4.0 (Blin et al. 2017). A total of 26 secondary metabolites biosynthesis clusters were identified in the $A$. alternata strain B3. These clusters comprise seven PKS clusters (annotated as T1PKS), three NRPS clusters, seven NRPS-like clusters, four terpene clusters, three PKS-NRPS cluster, one fungal-RiPP cluster, and one NRPS-indole cluster.

In the present study, we provide the draft genome sequence of $A$. alternata strain $\mathrm{B} 3$, a fungal species belonging to the family Pleosporaceae in the class Dothideomycetes. The sequence represents a useful source for future research on fungal comparative genomics studies.

Data availability. The whole genome shotgun project of $A$. alternata strain B3 has been deposited at DDBJ/ENA/GenBank under accession number JACBJF000000000 (BioProject PRJNA644072; BioSample SAMN15447420). The version described here is version JACBJF010000000. All the annotation data generated in this study have been deposited in the figshare repository at https://doi.org/10.6084/m9.figshare.12612797.

\section{Literature Cited}

Armenteros, J. J. A., Tsirigos, K. D., Sønderby, C. K., Petersen, T. N., Winther, O., Brunak, S., von Heijne, G., and Nielsen, H. 2019. SignalP 5.0 improves signal peptide predictions using deep neural networks. Nat. Biotechnol. 37:420-423.

Bankevich, A., Nurk, S., Antipov, D., Gurevich, A. A., Dvorkin, M., Kulikov, A. S., Lesin, V. M., Nikolenko, S. I., Pham, S., and Prjibelski, A. D. 2012. SPAdes: a new genome assembly algorithm and its applications to single-cell sequencing. J. Comput. Biol. 19:455-477.
Blin, K., Wolf, T., Chevrette, M. G., Lu, X., Schwalen, C. J., Kautsar, S. A., Suarez Duran, H. G., de Los Santos, E. L., Kim, H. U., and Nave, M. 2017. antiSMASH 4.0-improvements in chemistry prediction and gene cluster boundary identification. Nucleic Acids Res. 45 (W1):::W36-W41.

Bolger, A. M., Lohse, M., and Usadel, B. 2014. Trimmomatic: a flexible trimmer for Illumina sequence data. Bioinformatics 30:2114-2120.

Borodovsky, M., and Lomsadze, A. 2011. Eukaryotic gene prediction using GeneMark.hmm-E and GeneMark-ES. Curr. Protoc. Bioinformatics 4:1-10. 
Chan, P. P., and Lowe, T. M. 2019. tRNAscan-SE: Searching for tRNA genes in genomic sequences. Pages 1-14 in: Gene Prediction. Methods in Molecular Biology vol. 1962. M. Kollmar, ed. Humana, New York. .

Chen, M.-H., Chen, X.-J., Wang, M., Lin, L.-G., and Wang, Y.-T. 2016. Ophiopogon japonicus-A phytochemical, ethnomedicinal and pharmacological review. J. Ethnopharmacol. 181:193-213.

El-Gebali, S., Mistry, J., Bateman, A., Eddy, S. R., Luciani, A., Potter, S. C., Qureshi, M., Richardson, L. J., Salazar, G. A., and Smart, A. 2018. The Pfam protein families database in 2019. Nucleic Acids Res. 47 (D1):D427-D432.

Emanuelsson, O., Nielsen, H., Brunak, S., and Von Heijne, G. 2000. Predicting subcellular localization of proteins based on their N-terminal amino acid sequence. J. Mol. Biol. 300:1005-1016.

Fang, H., and Gough, J. 2013. DcGO: database of domain-centric ontologies on functions, phenotypes, diseases and more. Nucleic Acids Res. 41 (D1): D536-D544.

Fankhauser, N., and Mäser, P. 2005. Identification of GPI anchor attachment signals by a Kohonen self-organizing map. Bioinformatics 21:1846-1852.

Hoff, K. J., Lomsadze, A., Borodovsky, M., and Stanke, M. 2019. Whole-genome annotation with BRAKER. Pages 65-95 in: Gene Prediction. Methods in Molecular Biology vol. 1962. M. Kollmar, ed. Humana, New York.

Kim, D., Paggi, J. M., Park, C., Bennett, C., and Salzberg, S. L. 2019. Graph-based genome alignment and genotyping with HISAT2 and HISAT-genotype. Nat. Biotechnol. 37:907-915.

Krogh, A., Larsson, B., von Heijne, G., and Sonnhammer, E. L. 2001. Predicting transmembrane protein topology with a hidden Markov model: application to complete genomes. J. Mol. Biol. 305:567-580.

Kubicek, C. P., Starr, T. L., and Glass, N. L. 2014. Plant cell wall-degrading enzymes and their secretion in plant-pathogenic fungi. Annu. Rev. Phytopathol. 52: 427-451.
Lo Presti, L., Lanver, D., Schweizer, G., Tanaka, S., Liang, L., Tollot, M., Zuccaro, A. Reissmann, S., and Kahmann, R. 2015. Fungal effectors and plant susceptibility. Annu. Rev. Plant Biol. 66:513-545.

Möller, E. M., Bahnweg, G., Sandermann, H., and Geiger, H. H. 1992. A simple and efficient protocol for isolation of high molecular weight DNA from filamentous fungi, fruit bodies, and infected plant tissues. Nucleic Acids Res. 20: 6115-6116.

Simão, F. A., Waterhouse, R. M., loannidis, P., Kriventseva, E. V., and Zdobnov, E. M. 2015. BUSCO: assessing genome assembly and annotation completeness with single-copy orthologs. Bioinformatics 31:3210-3212.

Stanke, M., Diekhans, M., Baertsch, R., and Haussler, D. 2008. Using native and syntenically mapped cDNA alignments to improve de novo gene finding. Bioinformatics 24:637-644.

Thomma, B. P. 2003. Alternaria spp.: from general saprophyte to specific parasite. Mol. Plant Pathol. 4:225-236.

Tsuge, T., Harimoto, Y., Akimitsu, K., Ohtani, K., Kodama, M., Akagi, Y., Egusa, M., Yamamoto, M., and Otani, H. 2013. Host-selective toxins produced by the plant pathogenic fungus Alternaria alternata. FEMS Microbiol. Rev. 37:44-66.

Wang, M., Fu, H., Shen, X. X., Ruan, R., Rokas, A., and Li, H. 2019. Genomic features and evolution of the conditionally dispensable chromosome in the tangerine pathotype of Alternaria alternata. Mol. Plant Pathol. 20:1425-1438.

Woudenberg, J. H., Groenewald, J. Z., Binder, M., and Crous, P. W. 2013. Alternaria redefined. Stud. Mycol. 75:171-212.

Woudenberg, J. H., Seidl, M. F., Groenewald, J. Z., de Vries, M., Stielow, J. B., Thomma, B. P., and Crous, P. W. 2015. Alternaria section Alternaria: Species, formae speciales or pathotypes? Stud. Mycol. 82:1-21.

Zhang, H., Yohe, T., Huang, L., Entwistle, S., Wu, P., Yang, Z., Busk, P. K., Xu, Y., and Yin, Y. 2018. dbCAN2: a meta server for automated carbohydrate-active enzyme annotation. Nucleic Acids Res. 46 (W1):W95-W101. 